Journal of

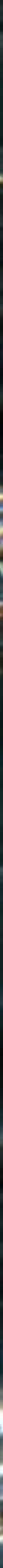

2

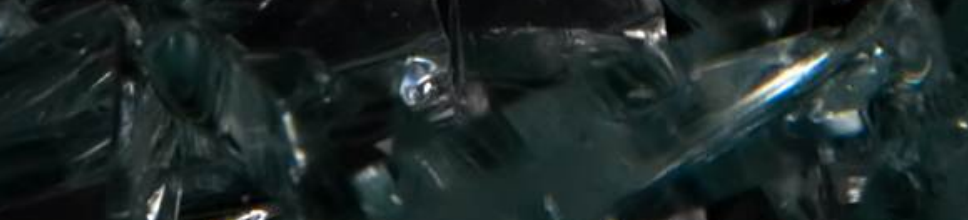

Volume 9 , No. 2

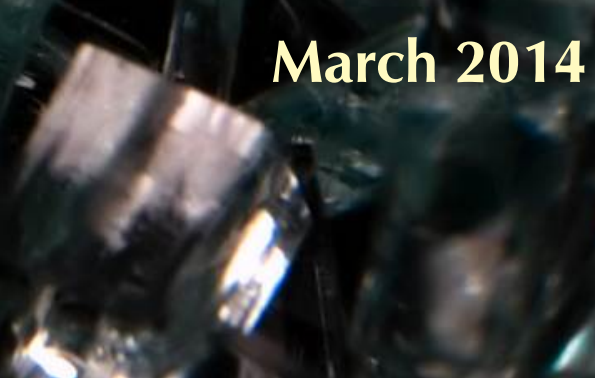




\title{
DYNAMIC RESPONSE OF TWIN LINED SHELLS DUE TO INCIDENT SEISMIC WAVES
}

\author{
J. P. DWIVEDi, V. P. Singh AND RADHA KRISHNA LAL
}

\begin{abstract}
The dynamic interaction of the twin shells subjected to seismic waves is investigated numerically. It is found that the three-dimensional response of twin shells may differ significantly from the two-dimensional response, and that through-soil interaction between the shells may also be significant.
\end{abstract}

\section{Introduction}

The dynamic response of buried pipelines and subway shells for structural stability during earthquakes is important when those structures can potentially be subjected to seismic ground motion. The amplification of seismic motions and stress concentrations may occur as a result of wave scattering around such structures. Mow and Pao [1973] were among the first to study wave diffraction around a cylindrical cavity in an infinite medium and the resulting stress concentration using wave function expansions. An analytic model was presented in [Trifunac 1972] to study horizontal polarized shear (SH) wave scattering at a semicylinder located at the boundary of a half-space. SH wave scattering for various topographies has also been studied in [Wong and Jennings 1975]. Chin et al. [1987] and Liu et al. [1991] studied the response of pipelines buried in back-filled trenches. In reality, many underground structures are constructed in close proximity and the interaction between such closely spaced structures may be significant [Okumura et al. 1992; Guan and Moore 1994]. The purpose of this paper is to investigate the threedimensional response of a pair of lined cylindrical cavities located in a full-space subjected to incident seismic waves.

\section{Governing equations}

The geometry of the problem is as shown in Figure 1. The parallel twin shells, denoted I and II, are deeply buried in a viscoelastic ground material described by the shear modulus $\mu$, mass density $\rho$, and Poisson's ratio $\sigma$. The material properties for the linings are described by the sets of variables $\mu_{\mathrm{I}}, \rho_{\mathrm{I}}$, and $\sigma_{\mathrm{I}}$ and $\mu_{\mathrm{II}}, \rho_{\mathrm{II}}$, and $\sigma_{\mathrm{II}}$. For convenience, the index indicating the medium or shell lining is omitted in (2-1)-(2-9), which are valid for each of these three different materials. The displacements and stresses in these solids can be expressed in terms of potentials $\phi, \psi$, and $\chi$, which satisfy the following wave equations of motion:

$$
\begin{aligned}
\nabla^{2} \phi+k_{\alpha}^{2} \phi & =0, \quad k_{\alpha}=\frac{\omega}{v_{L}}, \\
\nabla^{2} \psi+k_{\beta}^{2} \psi & =0,
\end{aligned}
$$

Radha Krishna Lal is the corresponding author.

Keywords: seismic waves, multiple successive reflections, shells. 


$$
\nabla^{2} \chi+k_{\beta}^{2} \chi=0, \quad k_{\beta}=\frac{\omega}{v_{L}}
$$

where $\omega$ is the excitation frequency, $v_{L}$ and $v_{T}$ denote the longitudinal and transverse wave velocities, and $\nabla^{2}$ is the Laplace operator in polar cylindrical coordinates.

The factor $e^{-i \omega\left(t-t_{0}\right)}$ is omitted in the text. The general solutions of (2-1), (2-2), and (2-3) take the following forms:

$$
\begin{aligned}
\phi(r, \theta, z)=\sum_{n=0}^{\infty} \phi_{n} & =\sum_{n=0}^{\infty} H_{n}\left(\alpha_{L} r\right)\left\{\begin{array}{l}
\cos n \theta \\
\sin n \theta
\end{array}\right\}\left(\left\{\begin{array}{l}
A \\
B
\end{array}\right\}_{n} e^{ \pm i \gamma_{L} z}\right) \\
& =\sum_{n=0}^{\infty} H_{n}\left(\alpha_{L} r\right)\left(A_{n} \cos n \theta+B_{n} \sin n \theta\right) e^{ \pm i \gamma_{L} z}, \\
\psi(r, \theta, z)=\sum_{n=0}^{\infty} \psi_{n} & =\sum_{n=0}^{\infty} H_{n}\left(\alpha_{T} r\right)\left\{\begin{array}{l}
\cos n \theta \\
\sin n \theta
\end{array}\right\}\left(\left\{\begin{array}{l}
C \\
D
\end{array}\right\}_{n} e^{ \pm i \gamma_{T} z}\right) \\
& =\sum_{n=0}^{\infty} H_{n}\left(\alpha_{T} r\right)\left(C_{n} \cos n \theta+D_{n} \sin n \theta\right) e^{ \pm i \gamma_{T} z}, \\
\chi(r, \theta, z)=\sum_{n=0}^{\infty} \chi_{n} & =\sum_{n=0}^{\infty} H_{n}\left(\alpha_{T} r\right)\left\{\begin{array}{l}
\cos n \theta \\
\sin n \theta
\end{array}\right\}\left(\left\{\begin{array}{l}
E \\
F
\end{array}\right\}_{n} e^{ \pm i \gamma_{T} z}\right) \\
& =\sum_{n=0}^{\infty} H_{n}\left(\alpha_{T} r\right)\left(E_{n} \cos n \theta+F_{n} \sin n \theta\right) e^{ \pm i \gamma_{T} z},
\end{aligned}
$$

where $H_{n}$ are the Bessel functions that satisfy the radiation conditions. Bessel functions of the first kind are used for the liners, and the outgoing wave in the medium is expressed in terms of Hankel functions

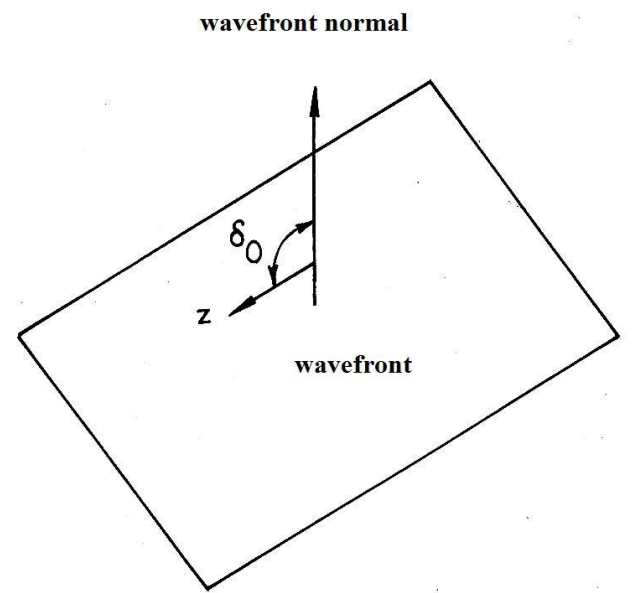

(a)

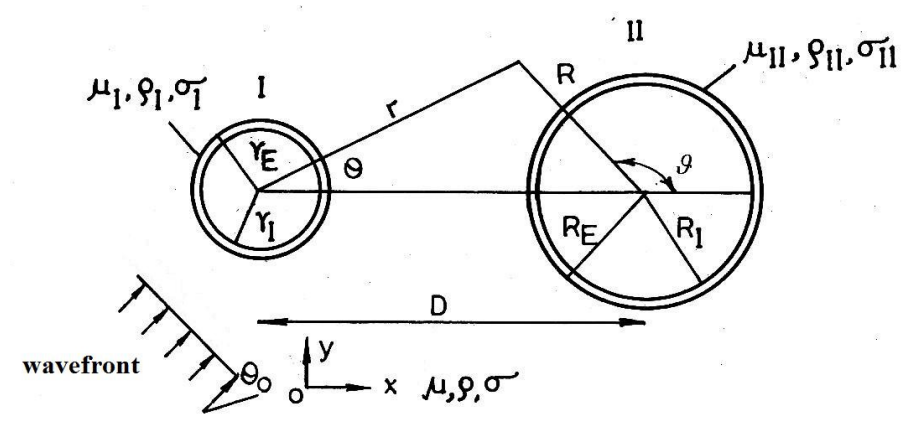

(b)

Figure 1. Geometry of the problem. 
of the first kind. The wave numbers $\alpha_{v}(v=L, T)$ are given by

$$
\begin{aligned}
& \alpha_{L}=\sqrt{\frac{\omega^{2}}{v_{L}^{2}-\gamma_{L}^{2}}}, \\
& \alpha_{T}=\sqrt{\frac{\omega^{2}}{v_{T}^{2}-\gamma_{T}^{2}}} .
\end{aligned}
$$

At the interface between the linings and the medium, the continuity and equilibrium conditions will be enforced:

$$
\begin{gathered}
\left(u_{r}^{(\mathrm{I})}, u_{\theta}^{(\mathrm{I})}, u_{z}^{(\mathrm{I})}\right)=\left(u_{r}, u_{\theta}, u_{z}\right), \\
\left(u_{r}^{(\mathrm{II})}, u_{\theta}^{(\mathrm{II})}, u_{z}^{(\mathrm{II})}\right)=\left(u_{r}, u_{\theta}, u_{z}\right), \\
\left(\sigma_{r r}^{(\mathrm{I})}, \sigma_{r \theta}^{(\mathrm{I})}, \sigma_{r z}^{(\mathrm{I})}\right)=\left(\sigma_{r r}, \sigma_{r \theta}, \sigma_{r z}\right), \\
\left(\sigma_{r r}^{(\mathrm{II})}, \sigma_{r \theta}^{(\mathrm{II})}, \sigma_{r z}^{(\mathrm{II})}\right)=\left(\sigma_{r r}, \sigma_{r \theta}, \sigma_{r z}\right) .
\end{gathered}
$$

For the problem specified in Figure 1, the wave field consists of the incident seismic waves and the waves scattered by shells I and II:

$$
\begin{aligned}
& \phi=\phi^{i}+\phi^{\mathrm{I}}(r, \theta, z)+\phi^{\mathrm{II}}(R, \vartheta, z), \\
& \psi=\psi^{i}+\psi^{\mathrm{I}}(r, \theta, z)+\psi^{\mathrm{II}}(R, \vartheta, z), \\
& \chi=\chi^{i}+\chi^{\mathrm{I}}(r, \theta, z)+\chi^{\mathrm{II}}(R, \vartheta, z),
\end{aligned}
$$

where the superscript $i$ denotes the incident seismic wave.

2.1. Coordinate system transform. In the numerical analysis, the interaction between the two shells is considered by coordinate system transformations (Figure 1(b)). The coordinate transformations from $(r, \theta)$ to $(R, \vartheta)$ and from $(R, \vartheta)$ to $(r, \theta)$ are [Watson 1944]

$$
\begin{aligned}
& H_{n}\left(\alpha_{v} r\right) \cos n \theta=\sum_{m=0}^{\infty} \frac{\epsilon_{m}}{2}\left(H_{n-m}\left(\alpha_{v} D\right) \pm(-1)^{m} H_{n+m}\left(\alpha_{v} D\right)\right) J_{m}\left(\alpha_{v} R\right) \cos m \vartheta \\
& H_{n}\left(\alpha_{v} r\right) \sin n \theta=\sum_{m=0}^{\infty} \frac{\epsilon_{m}}{2}\left(H_{n-m}\left(\alpha_{v} D\right) \pm(-1)^{m} H_{n+m}\left(\alpha_{v} D\right)\right) J_{m}\left(\alpha_{v} R\right) \sin m \vartheta
\end{aligned}
$$

and

$$
\begin{aligned}
& H_{n}\left(\alpha_{v} R\right) \cos n \vartheta=\sum_{m=0}^{\infty} \frac{\epsilon_{m}}{2}\left(H_{m-n}\left(\alpha_{v} D\right) \pm(-1)^{n} H_{m+n}\left(\alpha_{v} D\right)\right) J_{m}\left(\alpha_{v} r\right) \cos m \theta \\
& H_{n}\left(\alpha_{v} R\right) \sin n \vartheta=\sum_{m=0}^{\infty} \frac{\epsilon_{m}}{2}\left(H_{m-n}\left(\alpha_{v} D\right) \pm(-1)^{m} H_{m+n}\left(\alpha_{v} D\right)\right) J_{m}\left(\alpha_{v} r\right) \sin m \theta
\end{aligned}
$$

where

$$
\epsilon_{m}= \begin{cases}1 & \text { if } m=0 \\ 2 & \text { if } m=1,2, \ldots\end{cases}
$$


2.2. Incident seismic wave. The incident seismic wave can be expressed in terms of potentials. The incident $P$ wave and $S$ wave potentials can be written as

$$
\begin{aligned}
& \phi^{i}=\phi_{0} \exp \left(i k_{\alpha} \sin \delta_{0}\left(x \cos \theta_{0}\right)+y \sin \theta_{0}+i k_{\alpha} z \cos \delta_{0}-i \omega\left(t-t_{0}\right)\right), \\
& \psi^{i}=\psi_{0} \exp \left(i k_{\beta} \sin \delta_{0}\left(x \cos \theta_{0}\right)+y \sin \theta_{0}+i k_{\beta} z \cos \delta_{0}-i \omega\left(t-t_{0}\right)\right), \\
& \chi^{i}=\chi_{0} \exp \left(i k_{\beta} \sin \delta_{0}\left(x \cos \theta_{0}\right)+y \sin \theta_{0}+i k_{\beta} z \cos \delta_{0}-i \omega\left(t-t_{0}\right)\right),
\end{aligned}
$$

where $\delta_{0}$ is the angle of the wavefront normal to the $z$-axis (see Figure 1(a)), while $\theta_{0}$ is the angle that the normal of the intersection between the $X O Y$ plane and the wavefront makes with the $x$-axis (see Figure 1(b)).

Expressing the wave potentials in polar coordinates yields

$$
\begin{aligned}
\phi^{i} & =\phi_{0} \sum_{m=0}^{\infty} \frac{\epsilon_{m}}{2}(i)^{m} J_{m}\left(k_{\alpha} r \sin \delta_{0}\right)\left(A_{0, m} \cos m \theta+B_{0, m} \sin m \theta\right) \exp \left(i k_{\alpha} z \cos \delta_{0}-i \omega\left(t-t_{0}\right)\right), \\
\psi^{i} & =\psi_{0} \sum_{m=0}^{\infty} \frac{\epsilon_{m}}{2}(i)^{m} J_{m}\left(k_{\beta} r \sin \delta_{0}\right)\left(A_{0, m} \cos m \theta+B_{0, m} \sin m \theta\right) \exp \left(i k_{\beta} z \cos \delta_{0}-i \omega\left(t-t_{0}\right)\right), \\
\chi^{i} & =\chi_{0} \sum_{m=0}^{\infty} \frac{\epsilon_{m}}{2}(i)^{m} J_{m}\left(k_{\beta} r \sin \delta_{0}\right)\left(A_{0, m} \cos m \theta+B_{0, m} \sin m \theta\right) \exp \left(i k_{\beta} z \cos \delta_{0}-i \omega\left(t-t_{0}\right)\right),
\end{aligned}
$$

where

$$
A_{0, m}=\frac{\epsilon_{m}}{2}(i)^{m} \cos m \theta_{0}, \quad B_{0, m}=\frac{\epsilon_{m}}{2}(i)^{m} \sin m \theta_{0},
$$

and $J_{m}$ is a Bessel function of the first kind. Stresses $\tau_{r r}, \tau_{\theta \theta}$, and $\tau_{r \theta}$ and displacements $u_{r}$ and $u_{\theta}$ in the medium can be evaluated in terms of potentials (for example, in [Achenbach 1973]).

The potentials in (2-13)-(2-15) must satisfy the stress-free boundary conditions at the half-space and cavity surfaces:

$$
\tau_{x y}=\tau_{y y}=0 \text { at } y=0, \quad \tau_{r \theta}=\tau_{r r}=0 \text { at } r=r_{\mathrm{I}}, \quad \tau_{R \vartheta}=\tau_{R R}=0 \text { at } R=R_{\mathrm{I}},
$$

since $\psi^{i}, \chi^{i}$, and $\phi^{i}$ already satisfy the stress-free conditions at $y=0$.

2.3. Method of successive reflections. The approaches of Thiruvenkatachar and Viswanathan and Scheidl and Ziegler are used as follows:

(1) The incoming wave from shell II is assumed to be zero. Using the boundary conditions, the waves in the lining and the outgoing wave in the medium are determined at shell I.

(2) Using the boundary conditions, the waves in the lining and the outgoing wave in the medium at shell II are obtained. The incoming wave from shell I is then included using the coordinate transform of the outgoing wave at shell I.

(3) The waves in the lining and the outgoing wave in the medium at shell I are reevaluated with the incoming wave from shell II included.

(4) Steps (2) and (3) are repeated until the solution converges. 
2.4. Convergence. Following the convergence of the method of successive reflections by Thiruvenkatachar and Vishwanathan for wave scattering by a cylinder in a half-space, the convergence of the method is now examined for the specific case of two unlined cavities. Attention is focused on the response of cavity I as a result of the incident wave boundary conditions and wave reflection between the two cavities.

Therefore, with the incoming wave from cavity II excluded, a constant $k_{1}$ can be found for the upper bound of the outgoing wave coefficients due to unit incident waves:

$$
\left(\left|A_{n}^{\mathrm{I}}\right|,\left|B_{n}^{\mathrm{I}}\right|,\left|C_{n}^{\mathrm{I}}\right|,\left|D_{n}^{\mathrm{I}}\right|,\left|E_{n}^{\mathrm{I}}\right|,\left|F_{n}^{\mathrm{I}}\right|\right)<k_{\mathrm{I}} \frac{(r \alpha / 2)^{2 n}}{(n !)^{2}}(n+1), \quad n=0,1,2, \ldots,
$$

where $\alpha$ is the maximum of $\left|\alpha_{L}\right|$ and $\left|\alpha_{T}\right|$.

Substituting (2-29) into (2-16) and (2-17), it is then possible to find a positive number $k_{D}$ so that the following inequality holds for the incoming wave coefficients for cavity II:

$$
\left(\left|a_{m}^{\mathrm{II}}\right|,\left|b_{m}^{\mathrm{II}}\right|,\left|c_{m}^{\mathrm{II}}\right|,\left|d_{m}^{\mathrm{II}}\right|,\left|e_{m}^{\mathrm{II}}\right|,\left|f_{m}^{\mathrm{II}}\right|\right)<k_{\mathrm{I}} k_{D} \sum_{k=0}^{\infty} \epsilon_{m} \frac{\left(r_{i} \alpha / 2\right)^{2 k}(k+1)(m+k-1) !}{(D \alpha / 2)^{(m+k)}(k !)^{2}} .
$$

Similarly, the coefficients of the outgoing wave of cavity II are bounded as follows:

$$
\left(\left|A_{m}^{\mathrm{II}}\right|,\left|B_{m}^{\mathrm{II}}\right|,\left|C_{m}^{\mathrm{II}}\right|,\left|D_{m}^{\mathrm{II}}\right|,\left|E_{m}^{\mathrm{II}}\right|,\left|F_{m}^{\mathrm{II}}\right|\right)<k_{\mathrm{I}} k_{D} k_{\mathrm{II}} \sum_{k=0}^{\infty} \epsilon_{m} \frac{\left(r_{i} R_{i} \alpha^{2} / 2\right)^{2(k+m)}(m+k-1) !(k+1)(m+1)}{(D \alpha / 2)^{(m+k)}(k !)^{2}(m !)^{2}} .
$$

The incoming wave for cavity I can be obtained from the outgoing wave from cavity II using the coordinate transform once more:

$$
\begin{aligned}
\left(\left|a_{n}^{\mathrm{I}}\right|,\left|b_{n}^{\mathrm{I}}\right|,\left|c_{n}^{\mathrm{I}}\right|,\left|d_{n}^{\mathrm{I}}\right|,\left|e_{n}^{\mathrm{I}}\right|,\left|f_{n}^{\mathrm{I}}\right|\right) & \\
& <k_{\mathrm{I}} k_{D}^{2} k_{\mathrm{II}} \sum_{m=0}^{\infty} \epsilon_{n} \sum_{k=0}^{\infty} \epsilon_{m} \frac{\left(r_{i} R_{i} \alpha^{2} / 4\right)^{2(k+m)}(m+k-1) !(m+n-1) !(k+1)(m+1)}{(D \alpha / 2)^{(2 m+k+n)}(k !)^{2}(m !)^{2}} .
\end{aligned}
$$

It can be proved that the series defined by

$$
k_{D}^{n}=k_{D}^{2} \sum_{m=0}^{\infty} \epsilon_{n} \sum_{k=0}^{\infty} \epsilon_{m} \frac{\left(r_{i} R_{i} \alpha^{2} / 4\right)^{2(k+m)}(m+k-1) !(m+n-1) !(k+1)(m+1)}{(D \alpha / 2)^{(2 m+k+n)}(k !)^{2}(m !)^{2}}
$$

is uniformly convergent for a given $n$. Hence, the outgoing wave for cavity I (with incoming wave from cavity II included) is subject to

$$
\left(\left|A_{n}^{\mathrm{I}}\right|,\left|B_{n}^{\mathrm{I}}\right|,\left|C_{n}^{\mathrm{I}}\right|,\left|D_{n}^{\mathrm{I}}\right|,\left|E_{n}^{\mathrm{I}}\right|,\left|F_{n}^{\mathrm{I}}\right|\right)<k_{\mathrm{I}}\left(1+k_{1} k_{D}^{n} k_{\mathrm{II}}\right) \frac{\left(r_{i} \alpha^{2} / 2\right)^{2 n}}{(n !)^{2}} .
$$

Following $m$ iterations of the method of successive reflections, the outgoing wave of cavity I gives

$$
\left(\left|A_{n}^{\mathrm{I}}\right|,\left|B_{n}^{\mathrm{I}}\right|,\left|C_{n}^{\mathrm{I}}\right|,\left|D_{n}^{\mathrm{I}}\right|,\left|E_{n}^{\mathrm{I}}\right|,\left|F_{n}^{\mathrm{I}}\right|\right)<\sum_{j=0}^{\infty} k_{\mathrm{I}}\left(k_{1} k_{D}^{n} k_{\mathrm{II}}\right) \frac{\left(r_{i} \alpha / 2\right)^{2 n}}{(n !)^{2}}=k_{\mathrm{I}} \frac{1-\left(k_{\mathrm{I}} k_{D}^{n} k_{\mathrm{II}}\right)^{(m+1)}}{1-k_{\mathrm{I}} k_{D}^{n} k_{\mathrm{II}}} \frac{\left(r_{i} \alpha / 2\right)^{2 n}}{(n !)^{2}} .
$$

The method of successive reflections therefore converges provided $k_{\mathrm{I}} k_{D}^{n} k_{\mathrm{II}}<1$. The spacing $D$ between the two cavities in (2-33) can be changed for a given $r_{i}$ and $R_{i}$ so that $k_{\mathrm{I}} k_{D}^{n} k_{\mathrm{II}}<1$. Therefore the 
procedure converges if either of the following two conditions is satisfied:

(1) The wavelength is long enough or there is sufficient spacing between the two cavities.

(2) The $k_{\mathrm{I}} k_{D}^{n} k_{\mathrm{II}}<1$ values for the dominant terms (for example, $\left.n=2\right)$ are particularly small $\left(k_{\mathrm{I}} k_{D}^{n} k_{\mathrm{II}} \ll 1\right.$ ).

It is remarkable to note that, in some specific problems, the expected divergence may also be removed by a large number of iterations of the technique applied in this paper.

\section{Numerical study}

3.1. Geometry. The response of the twin lined shells to seismic ground motion is clearly a function of a considerable number of geometrical and material properties. In order to simplify this study, the response will be examined for shells of equal radius $\left(r_{i}=R_{i}\right)$ and with identical linings $\left(r_{e}=R_{e}, \mu=\mu_{\mathrm{I}}=\mu_{\mathrm{II}}\right)$. The Poisson's ratios for the medium and liners are 0.30 and 0.35 , respectively. Furthermore, the center-tocenter spacing of the shells will be taken as $D=3.50 r_{i}, 4.50 r_{i}$, or $\infty$, and the liner thickness, $r_{e}-r_{i}=r_{i} / 8$, and the incident seismic waves examined will be parallel to either the $\operatorname{YOZ}\left(\theta_{0}=\pi / 2\right)$ or the $\operatorname{XOZ}\left(\theta_{0}=0\right)$ plane. The two shells have axes parallel to $z$, so that for $\theta_{0}=\pi / 2$ the shells have symmetric response about the $Y O Z$ plane and only one shell response needs to be reported. Alternatively, for $\theta_{0}=0$ the shell responses are different but symmetric about the horizontal diameters. For all the results presented, the displacements and stresses are normalized by $k_{\beta}$ and $\mu k_{\beta}^{2}$, respectively.

3.2. Incident $S$ wave: interaction between the shells. To examine the interaction of the two shells, Figure 2 shows predictions of the normalized hoop stress for shells at three different spacings, $D / r_{i}=$ $3.50,4.50$, and $\infty$, and for two different incident angles, $\delta_{0}=\pi / 2$ and $\delta_{0}=\pi / 3$. To determine the effect of the frequency of the seismic ground motion, a range of normalized vibration frequencies $a_{0}=\omega r_{i} / v_{T}$ is considered.
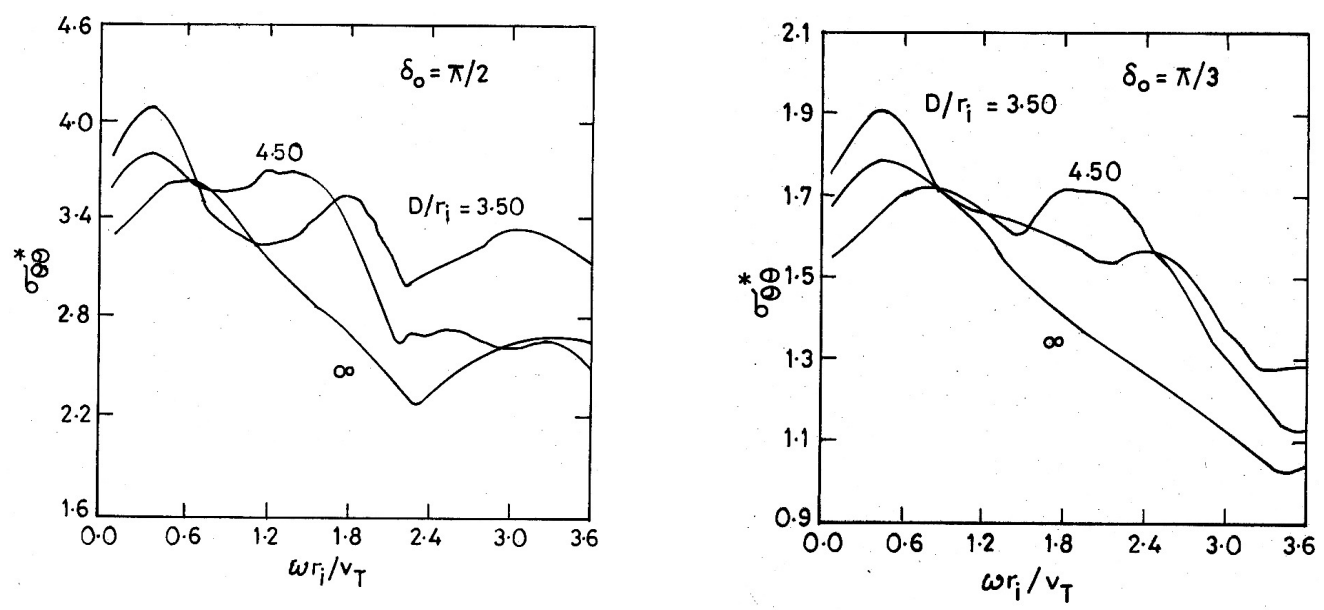

Figure 2. Maximum stress versus normalized frequency: response to incident $S$ waves at the inside surface of the liner, at position $z=0\left(\theta_{0}=\pi / 2, \mu / \mu_{m}=3\right)$ for $\delta_{0}=\pi / 2$ (left) and $\delta_{0}=\pi / 3$ (right). 

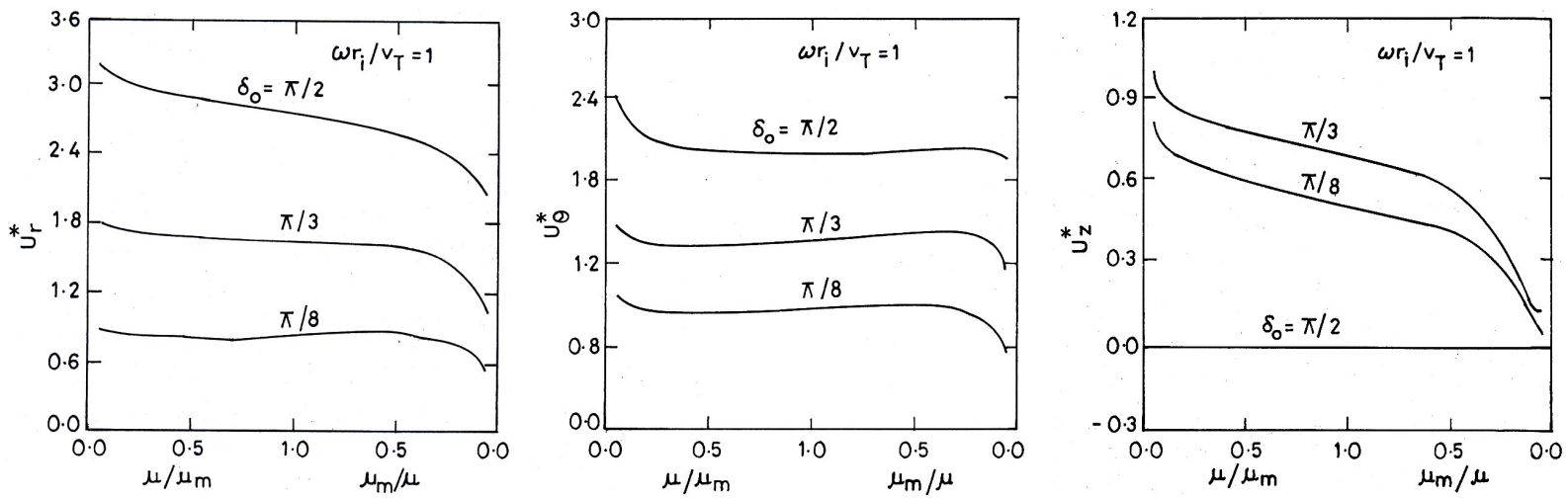

Figure 3. Maximum displacements $U_{r}^{*}$ (left), $U_{\theta}^{*}$ (middle), and $U_{Z}^{*}$ (right) versus normalized frequency: response to incident $S$ waves at the outside surface of the liner, at position $z=0\left(\theta_{0}=0, D / r_{i}=3.50\right)$.

At low vibration frequency, $a_{0}<0.5$, the shell-to-shell interaction for all three shell spacings is insignificant. As normalized frequency $a_{0}$ increases, however, the shell response becomes significantly influenced by the spacing. For $0.5<a_{0}<2.5$, the hoop stress $\sigma_{\theta \theta}^{*}$ is up to $35 \%$ less for an isolated shell $D / r_{i}=\infty$ than for $D / r_{i}=3.50,4.50$. For shells with $D<3 r_{i}$, the increase in hoop stress due to through-soil interaction is expected to be even more important.

3.3. Incident $S$ wave: influence of lining modulus. To examine the influence of lining design, Figure 3 shows solutions for the maximum normalized displacements $U_{r}^{*}, U_{\theta}^{*}$, and $U_{z}^{*}$ for the specific normalized frequency $a_{0}=1$, for a range of modular ratios $\mu / \mu_{m}$ and for three different incident angles $\delta_{0}$. Figure 4 shows solutions for the maximum normalized stresses $\sigma_{\theta \theta}^{*}$ and $\sigma_{\theta z}^{*}$ for the specific normalized frequency $a_{0}=1$, for a range of modular ratios $\mu / \mu_{m}$ and for three different incident angles $\delta_{0}$.
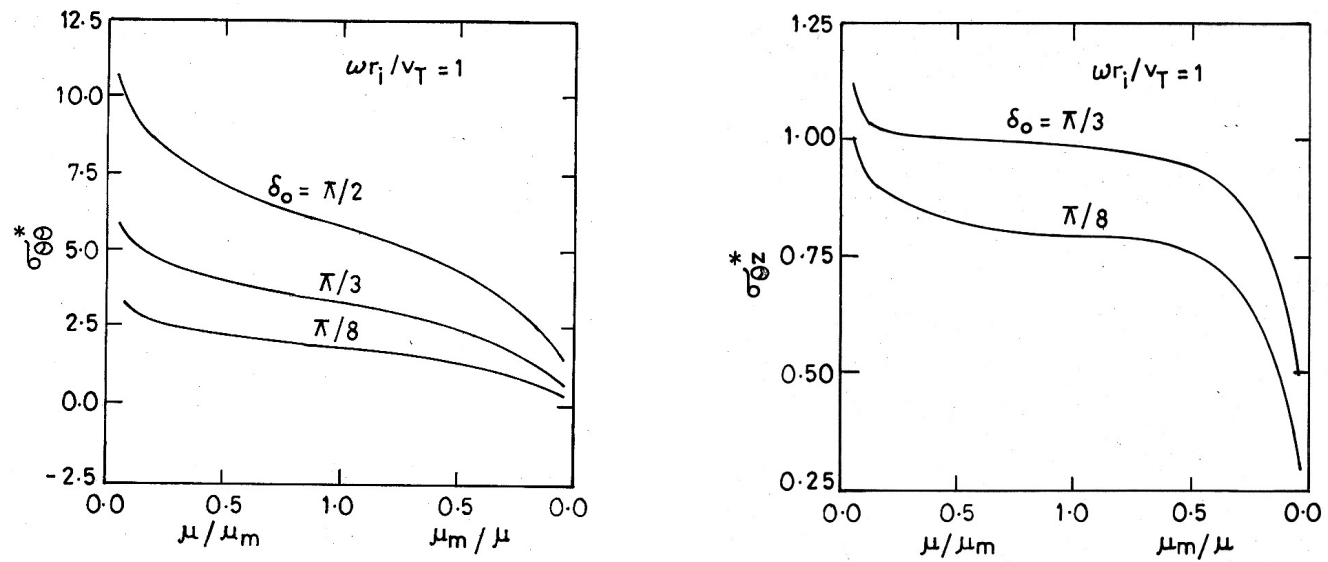

Figure 4. Maximum normalized stresses $\sigma_{\theta \theta}^{*}$ (left) and $\sigma_{\theta z}^{*}$ (right) versus normalized frequency: response to incident $S$ waves at the inside surface of the liner, at position $z=0\left(\theta_{0}=0, D / r_{i}=3.50\right)$. 

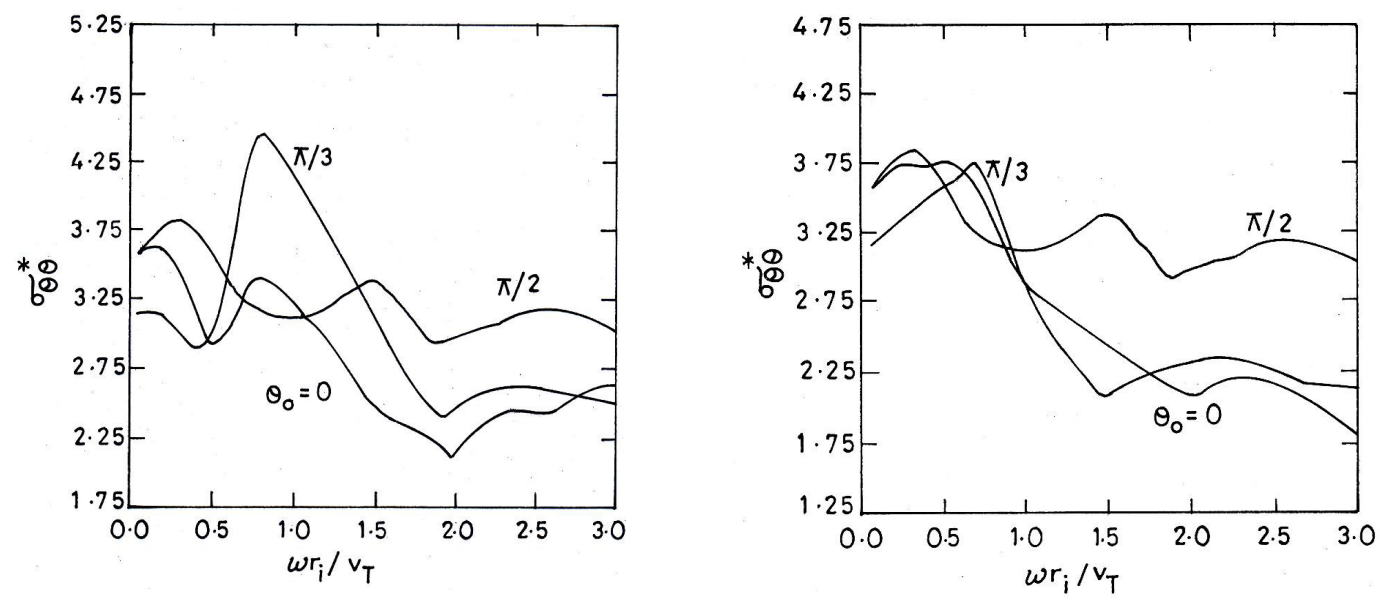

Figure 5. Maximum stress versus normalized frequency: response to incident $S$ waves at the inside surface of the liner, at position $z=0\left(\delta_{0}=\pi / 2, \mu / \mu_{m}=3\right)$ for shells I (left) and II (right).

The normalized radial and circumferential displacements are the greatest responses under plane strain conditions, $\delta_{0}=\pi / 2$. They peak at low modulus ratio, $\mu / \mu_{m}$, but remain nearly constant when $\mu / \mu_{m}$ ranges from 0.25 to 4 . Normalized longitudinal displacement also decreases monotonically as $\mu / \mu_{m}$ increases, but is generally more sensitive to any changes in the modular ratio.

Normalized stress amplitudes $\sigma_{\theta \theta}^{*}$ and $\sigma_{\theta z}^{*}$ at the inner surface of the lining all steadily decrease as the surrounding modulus, $\mu_{m}$, decreases relative to that of liners $\mu$. All values tend towards zero as modular ratio $\mu_{m} / \mu$ approaches zero. As noted for the normalized displacement, the two-dimensional loading condition $\delta_{0}=\pi / 2$ induces the greatest stress. Circumferential stresses $\sigma_{\theta \theta}^{*}$ are largest in magnitude.

3.4. Incident $S$ wave: influence of incident angle $\boldsymbol{\theta}_{0}$. The behavior of the two shells is now examined under the plane strain condition $\left(\delta_{0}=\pi / 2\right)$ with incident angles $\theta_{0}=0, \pi / 3$, and $\pi / 2$, and a range of vibration frequencies. The shells are spaced at distance $D / r_{i}=3.50$ and have a lining modulus three times that of the ground medium $\left(\mu / \mu_{m}=3\right)$. Figure 5 shows the amplitude of the normalized stress $\sigma_{\theta \theta}^{*}$ for shells I and II.

For an isolated shell, the response is independent of the incident angle $\theta_{0}$. However, for these closely spaced shells, the response is significantly affected by the orientation of the incoming wave. Firstly, Figure 5 clearly shows that the effect of the incident angle $\theta_{0}$ is frequency dependent. The circumferential stress $\sigma_{\theta \theta}^{*}$ of shell I has the greatest amplitude when $\theta_{0}=\pi / 3$ at the normalized frequency $a_{0}=0.8$. For larger values of normalized frequency, $a_{0}>1.5$, the responses are less frequency dependent and the vertically moving seismic wave $\left(\theta_{0}=\pi / 2\right)$ induces the highest hoop stresses in both shells. For shell II the peak stress occurs when $0.2<a_{0}<0.8$, but is not quite as great and is less dependent on $\theta_{0}$.

3.5. Incident $\boldsymbol{P}$ wave. Figure 6 shows normalized radial, circumferential, and longitudinal displacements for $\theta=\pi / 2$, for three incident wave angles $\delta_{0}$ and a range of the modular ratios. The trends are similar to those observed earlier for shell response to shear waves, but with stress and displacement amplitudes somewhat smaller. The response increases as the modular ratio increases. Figure 7 shows 

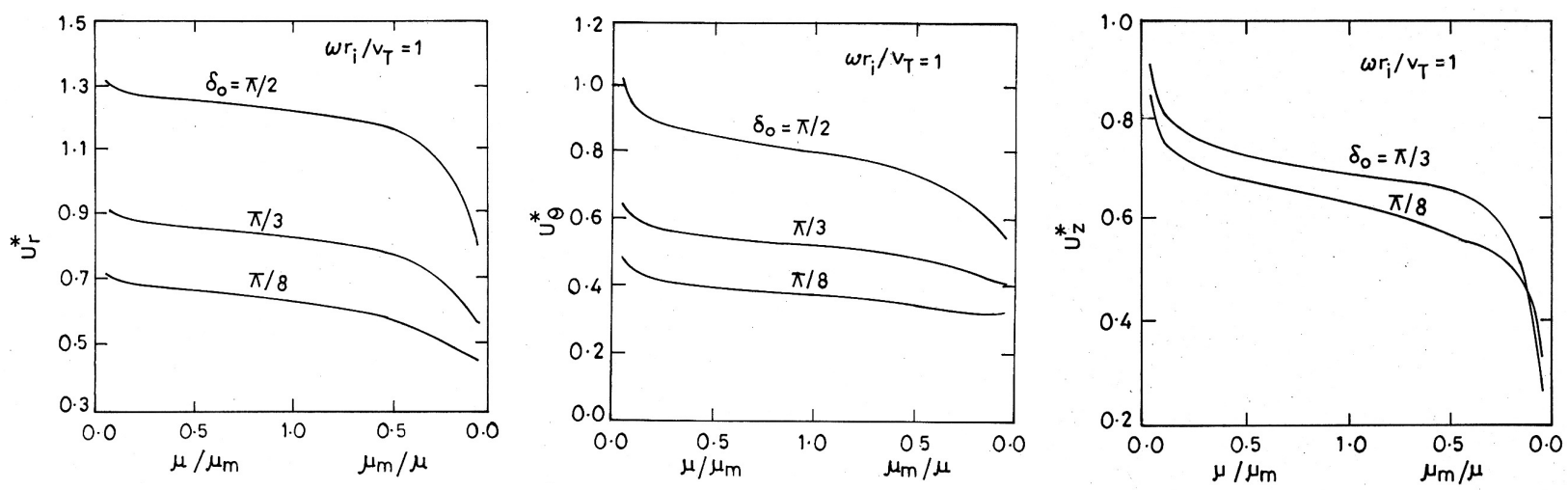

Figure 6. Maximum displacements $U_{r}^{*}$ (left), $U_{\theta}^{*}$ (middle), and $U_{Z}^{*}$ (right) versus normalized frequency: response to incident $P$ waves at the outside surface of the liner, at position $z=0\left(\theta_{0}=\pi / 2, D / r_{i}=3.50\right)$.
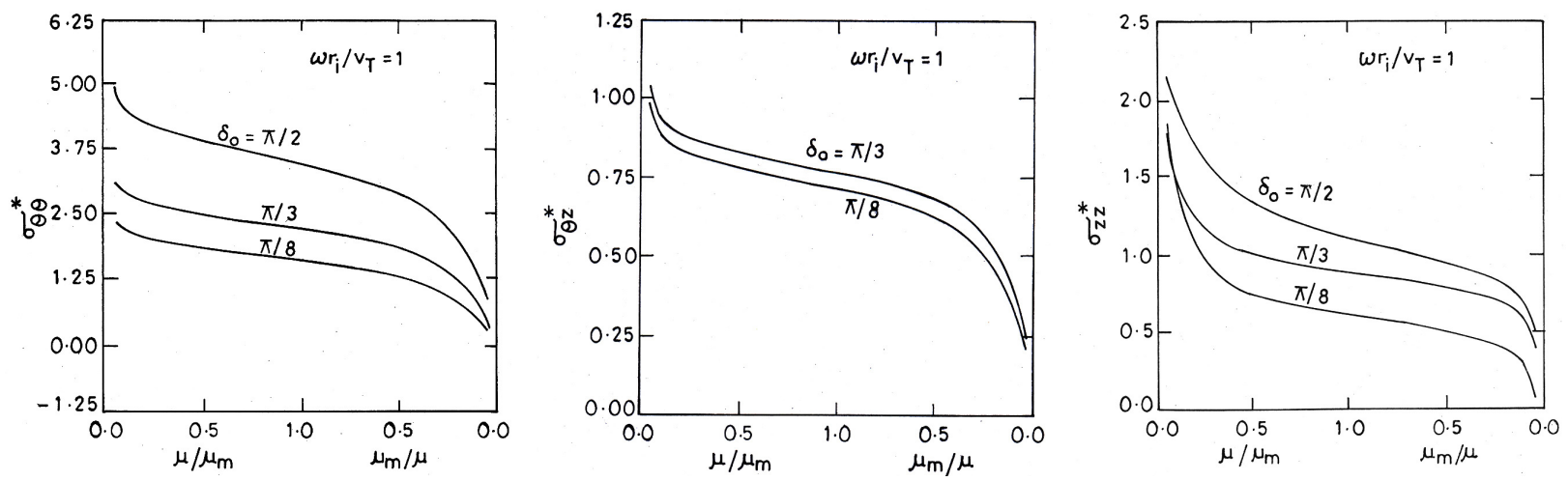

Figure 7. Maximum stresses $\sigma_{\theta \theta}^{*}$ (left), $\sigma_{\theta z}^{*}$ (middle), and $\sigma_{z z}^{*}$ (right) versus normalized frequency; response to incident $P$ waves at the inside surface of the liner, at position $z=0\left(\theta_{0}=0, D / r_{i}=3.50\right)$.

solutions for maximum normalized stresses $\sigma_{\theta \theta}^{*}, \sigma_{\theta z}^{*}$, and $\sigma_{z z}^{*}$ for the specific normalized frequency $a_{0}=1$, for a range of modular ratios $\mu / \mu_{m}$ and three different incident angles $\delta_{0}$. Compared to the normalized displacement amplitudes, the stresses are more sensitive to changes in the modular ratios.

3.6. Incident $P$ wave: interaction between the shells. The interaction between the shells is shown in Figure 8 different angles, $\delta_{0}=\pi / 2$ and $\pi / 4$. Unlike the prediction made for the shell response to an $S$ wave, the hoop stress $\sigma_{\theta \theta}^{*}$ decreases rapidly as the frequency is increased. The through-soil interaction between the shells is not as significant as it is for an incident $S$ wave. At most frequencies, the stress predictions for the closely spaced shells are higher than those for an isolated shell, though the difference is not very significant.

3.7. Incident $\boldsymbol{P}$ wave: influence of incident angle. Figure 9 shows the hoop stress response for shells I and II. The peak hoop stress is produced for an incident angle $\delta_{0}=0$ and a normalized frequency $a_{0}=1$. 

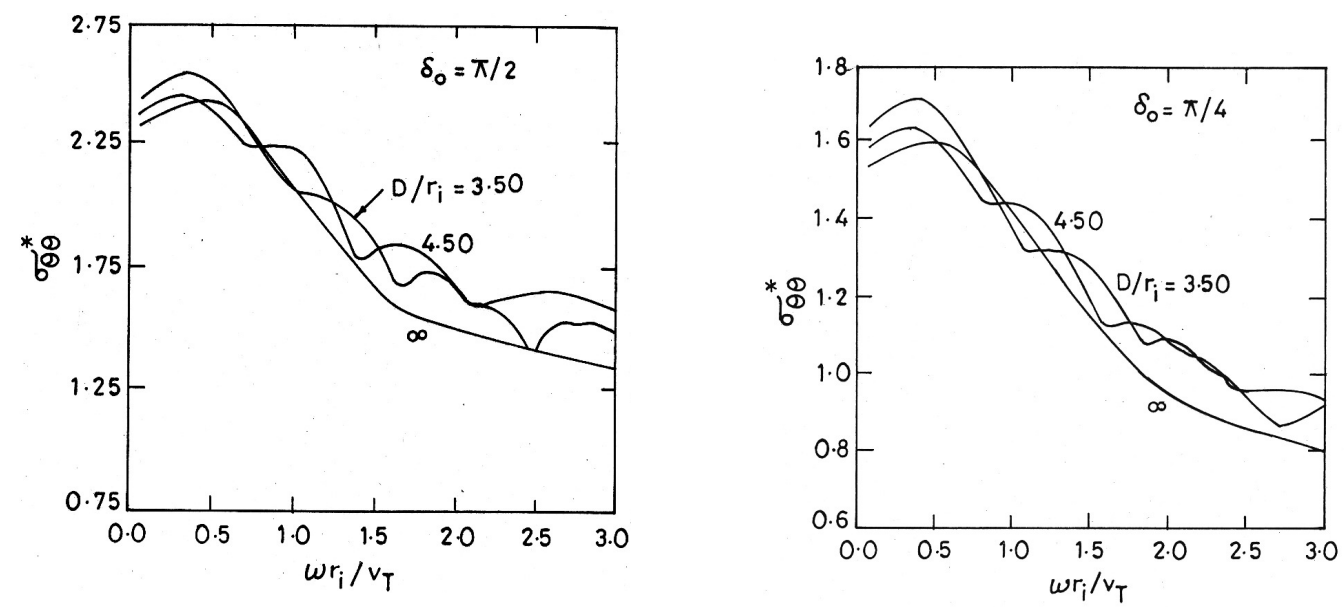

Figure 8. Maximum stress versus normalized frequency: response to incident $P$ waves at the inside surface of the liner, at position $z=0\left(\theta_{0}=\pi / 2, \mu / \mu_{m}=3\right)$ for $\delta_{0}=\pi / 2$ (left) and $\delta_{0}=\pi / 4$ (right).
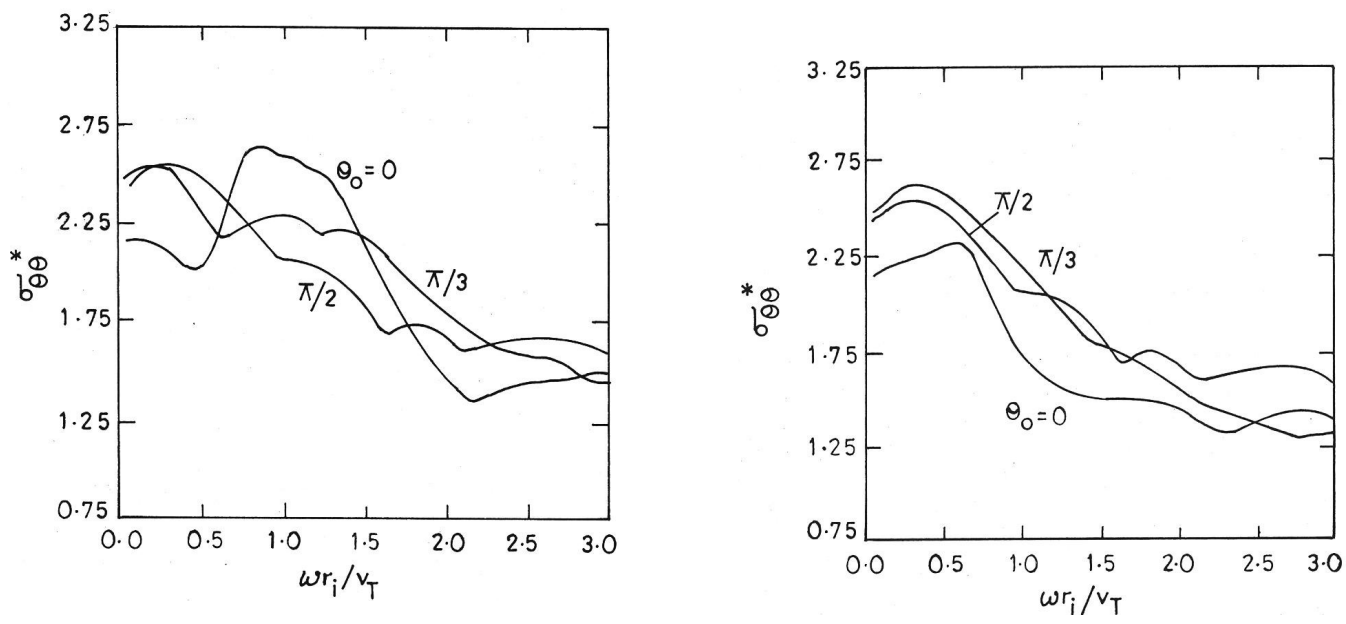

Figure 9. Maximum stress versus normalized frequency; response to incident $P$ waves at the inside surface of the liner, at position $z=0\left(\delta_{0}=\pi / 2, \mu / \mu_{m}=3\right.$, and $\left.D / r_{i}=3.50\right)$ for shells I (left) and II (right).

However, less response is induced in shell II for incident angle $\theta_{0}=0$ since it is shielded by shell I (shell II is in the "shadow" of shell I).

\section{Acknowledgments}

The authors are grateful to the referee for valuable suggestions and comments for the improvement of this paper. The authors also thank Prof. P. C. Upadhyay, former head of the Department of Mechanical Engineering, Indian Institute of Technology (Banaras Hindu University), Varanasi, for his kind help in the preparation of this paper. 


\section{References}

[Achenbach 1973] J. D. Achenbach, Wave propagation in elastic solids, North-Holland Series in Applied Mathematics and Mechanics 16, North-Holland, Amsterdam, 1973.

[Chin et al. 1987] Y. F. Chin, R. K. N. D. Rajapakse, A. H. Shah, and S. K. Datta, "Dynamics of buried pipes in back-filled trench", Soil Dyn. Earthq. Eng. 6:3 (1987), 158-163.

[Guan and Moore 1994] F. Guan and I. D. Moore, "Three-dimensional dynamic response of twin cavities due to traveling loads", J. Eng. Mech. (ASCE) 120:3 (1994), 637-651.

[Liu et al. 1991] S. W. Liu, A. H. Shah, S. K. Datta, and K. R. Khair, "Dynamic response of pipelines buried in back-filled trenches", J. Press. Vessel Technol. (ASME) 113:3 (1991), 429-436.

[Mow and Pao 1973] C.-C. Mow and Y.-H. Pao, The diffraction of elastic waves and dynamic stress concentrations, Crane Russak, New York, 1973.

[Okumura et al. 1992] T. Okumura, N. Takewaki, K. Shimizu, and K. Fukutake, "Dynamic response of twin circular tunnels during earthquakes", pp. 181-191 in Proceedings of the 4th US-Japan workshop on earthquake disaster prevention for lifeline systems (Los Angeles, 1991), edited by R. T. Equchi, NIST Special Publication 840, US Department of Commerce, Gaithersburg, MD, 1992.

[Trifunac 1972] M. D. Trifunac, "Scattering of plane SH waves by a semi-cylindrical canyon", Earthq. Eng. Struct. Dyn. 1:3 (1972), 267-281.

[Watson 1944] G. N. Watson, A treatise on the theory of Bessel functions, Cambridge University, 1944.

[Wong and Jennings 1975] H. L. Wong and P. C. Jennings, "Effects of canyon topography on strong ground motion", Bull. Seismol. Soc. Am. 65:5 (1975), 1239-1257.

Received 7 Sep 2013. Revised 28 Dec 2013. Accepted 26 Jan 2014.

J. P. DWIVEDI: jpd@bhu.ac.in

Department of Mechanical Engineering, Indian Institute of Technology (Banaras Hindu University), Varanasi 221005, India

V. P. SINGH: vp_singh56@yahoo.co.in

Department of Mechanical Engineering, Indian Institute of Technology (Banaras Hindu University), Varanasi 221005, India

RADHA KRISHNA LAL: radhakrishna773@gmail.com

Department of Mechanical Engineering, Indian Institute of Technology (Banaras Hindu University), Varanasi 221005, India 


\title{
JOURNAL OF MECHANICS OF MATERIALS AND STRUCTURES
}

\author{
msp.org/jomms
}

\author{
Founded by Charles R. Steele and Marie-Louise Steele
}

EDITORIAL BOARD

ADAIR R. AGUIAR

KATIA BERTOLDI

University of São Paulo at São Carlos, Brazil

DAVIDE BIGONI

Harvard University, USA

IWONA JASIUK

University of Trento, Italy

Thomas J. PENCE

University of Illinois at Urbana-Champaign, USA

YASUHIDE SHINDO

Michigan State University, USA

DAVID STEIGMANN

Tohoku University, Japan

University of California at Berkeley

\section{ADVISORY BOARD}

J. P. CARTER University of Sydney, Australia

R. M. Christensen Stanford University, USA

G. M. L. GLADWELL University of Waterloo, Canada

D. H. Hodges Georgia Institute of Technology, USA

J. HUTCHINSON Harvard University, USA

C. HwU National Cheng Kung University, Taiwan

B. L. KARIHALOO University of Wales, UK

Y. Y. KIM Seoul National University, Republic of Korea

Z. Mroz Academy of Science, Poland

D. PAMPlonA Universidade Católica do Rio de Janeiro, Brazil

M. B. RubiN Technion, Haifa, Israel

A. N. SHUPIKOV Ukrainian Academy of Sciences, Ukraine

T. TARNAI University Budapest, Hungary

F. Y. M. WAN University of California, Irvine, USA

P. WRIGGERS Universität Hannover, Germany

W. YANG Tsinghua University, China

F. ZIEGLER Technische Universität Wien, Austria

PRODUCTION production@msp.org

SILVIO LEVY Scientific Editor

Cover photo: Mando Gomez, www.mandolux.com

See msp.org/jomms for submission guidelines.

JoMMS (ISSN 1559-3959) at Mathematical Sciences Publishers, 798 Evans Hall \#6840, c/o University of California, Berkeley, CA 94720-3840, is published in 10 issues a year. The subscription price for 2014 is US \$555/year for the electronic version, and $\$ 710 /$ year (+\$60, if shipping outside the US) for print and electronic. Subscriptions, requests for back issues, and changes of address should be sent to MSP.

JoMMS peer-review and production is managed by EditFLow ${ }^{\circledR}$ from Mathematical Sciences Publishers.

PUBLISHED BY

mathematical sciences publishers

nonprofit scientific publishing

http://msp.org/

(C) 2014 Mathematical Sciences Publishers 


\title{
Journal of Mechanics of Materials and Structures
}

\author{
Volume 9, No. $2 \quad$ March 2014
}

Plane waves at the boundary of two micropolar thermoelastic solids with distinct conductive and thermodynamic temperatures

Rajneesh Kumar, Mandeep Kaur and Satish C. RaJvanshi

Dynamic compression of square tube cellular structures RYAN L. HOLLOMAN, KaRTHIKEyan Kandan, VikRam DeshPande and HaYdn N. G. WadLey

Dynamic response of twin lined shells due to incident seismic waves

J. P. DWIVEdi, V. P. Singh and RADHA KRISHNA LAL 183

Solutions of the von Kármán plate equations by a Galerkin method, without inverting the tangent stiffiness matrix.

Honghua Dai, Xiaokuí Yue and Satya N.Atluri 195

Bimaterial lattices with anisotropic thermal expansion

Marina M. Toropova and Craig A. Steeves

Origin and effect of nonlocality in a composite STEWART A. Silling 